\title{
Research on Real Time Power System Dynamic Monitoring Master Station System Test and Evaluation
}

\author{
Kun Zhao, Sheng Liu, Zhi Cai, Yang Bai, Yansheng Lang, Qiang Li \\ China Electric Power Research Institute \\ Beijing, China
}

\begin{abstract}
Phasor measurement technology developed rapidly in CHINA, Real time power system dynamic monitoring master station system were built widely in inter-regional power system and provincial power system, With WAMS system of practical ,WAMS master station test specification making is particularly important .WAMS master station system is evaluated from system hardware system software of the basic functions and other application functions, the methods and evaluation functions are designed. The evaluation functions of WAMS master station system is designed based on the three levels of content. To the stability and the reliability, the evaluation methods and functions are designed for a certain period. At last based on the above evaluation methods and functions, aquantitative indicators are provided for the WAMS master station system operation state.
\end{abstract}

Keywords- phasor measurements, WAMS master station, evaluation methods, test specification

\section{INTRODUCTION}

Since the 1990s,Phasor Measurement Unit(PMU) technology provided a new technical scheme for dynamic monitoring and accident analysis of power grid. main characteristic of PMU is the instantaneity which identified with the GPS clock synchronization ${ }^{[1]}$.Wide Area Measurement System(WMAS) which is signed by application of PMU gets a different type of pilots applications $^{[2-7]}$.With the development of modern large-scale grid technology, dynamic monitoring and control system based on PMU technology have become the hot spots within grid real-time dynamic security and control technology area. At present,2500 sets of PMU have been put into operation in our country, which has the world's most PMUs. Around 2003, WAMS is established in each network-provincial power scheduling center, such as country scheduling, north china scheduling, north-east, east china, north-west,

JiangSu, Guangdong. power grid dynamic monitoring ${ }^{[8-10]}$, low frequency oscillation monitoring and analysis ${ }^{[11,12]}$, disturbance identification ${ }^{[13,14]}$, power station an time frequency modulation evaluation ${ }^{[15]}$, power system model and parameter checking etc were put into operation based on PMU data.

Along with the PMUs, WAMS installed and put into use, related standard operations have been launched quickly. In march 2003,the national power scheduling center promulgated $<<$ wide area real time measure system technical specification for power system $>>$, which was revised in October 2004.A series of standard operations and test specification were carried out wildly, in order to regulate technical requirements of PMU sub-station and WAMS master system. However, the corresponding evaluation research is very few. how are the WAMS master system practical operation level, performance of the hardware and software? There are no a method to evaluate it. Based on practical level of WAMS master system, three levels evaluation method is designed for the WAMS master system, functionality and performance of the master system hardware and software are evaluated by the method, at last, a quantitative indicators is proposed.

\section{WAMS MASTER SYSTEM}

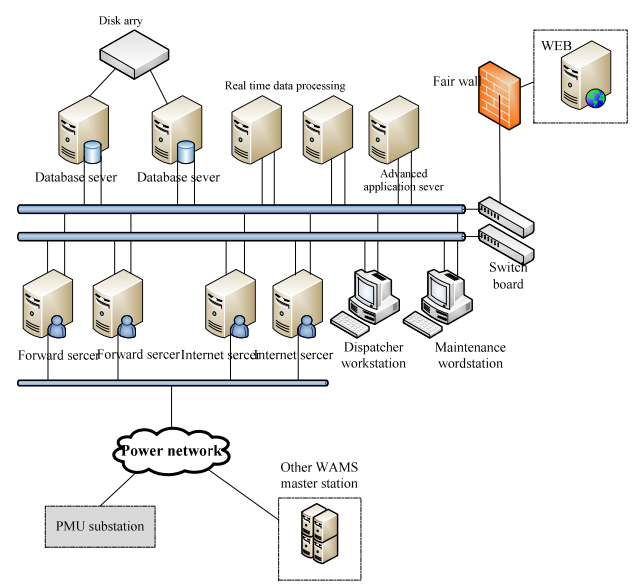

Figure 1. WAMS master system deploy

WAMS system is generally installed at each network-provincial power scheduling in china. WAMS, which is based on PMU with synchronous phasor acquisition technology, mainly consists three parts. Firstly, devices of data collection or PDC, secondly, message transfer part based on power communication network, Thirdly, master station data processing and application using at power grid scheduling. The processes with collection, transform, processing, application of PMU data, consists of the three links.

\section{WAMS MASTER SYSTEM TEST AND EVALUATION THINKING}

The practical test specification and evaluation scheme of WAMS master system is designed. The test content, which is divided into three classes, includes system basic 
hardware, system basic software function, other system and application function. The three content of the thinking is executed in order(see figure 2), evaluation steps as follows:

a) WAMS master system hardware evaluation. The major hardware and environment of master system are evaluated whether them meet the test standards.

b) WAMS master system basic software function evaluation. The software function is tested and evaluated, in order to ensure the availability of the master system.

c) system and application function evaluation. All of the system function, application function, performance are evaluated, in order to judge how is the practical level of WAMS master system.

d) WAMS master system is tested and evaluated, and the evaluation result means performance level of master system operating in a time range.

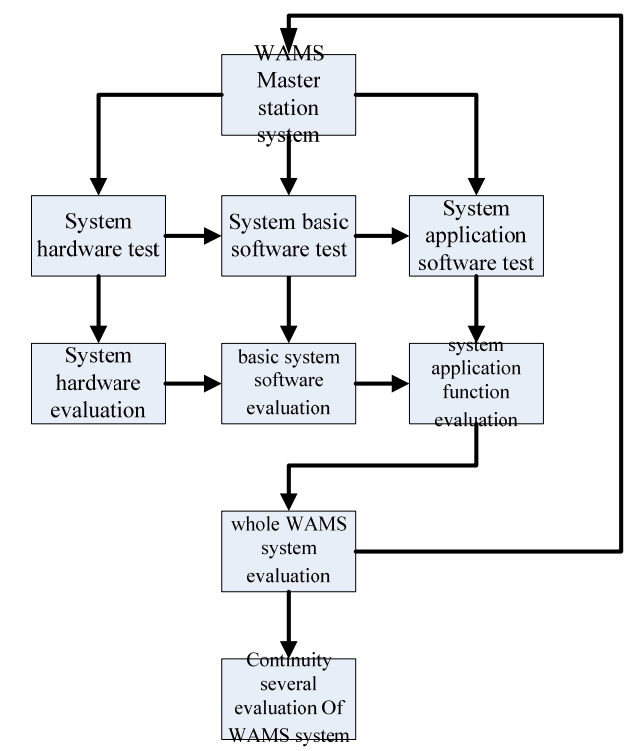

Figure 2. WAMS master system test and evaluation flow chart

\section{WAMS MASTER SYSTEM EVALUATION METHOD}

A. System hardware evaluation method

system hardware is footstone, based on that the WAMS system can operate normally, and hardware performance and environment are the factors which restrict the performance development of the whole master system.

Basic system hardware includes previa, data collection server,real-time server, historical data server,graphical monitoring workstation, power source etc.System hardware,the basic of the master station system,is influenced by temperature ,humidity,atmospheric pressure,risk of explosion,corrosive gas,mycete etc.

set of basic system hardware as follows:

$$
D E V=\left\{\operatorname{dev}_{1}, \operatorname{dev}_{2}, \ldots, \operatorname{dev}_{i}, \ldots, \operatorname{dev}_{n 1}\right\}
$$

set of influence factors as follows:

$$
I N L=\left\{\text { inl }_{1}, \text { inl }_{2}, \ldots, \text { inl }_{l}, \ldots, \text { inl }_{m 1}\right\}
$$

evaluation function designed as follows:

$$
\begin{aligned}
& \text { ase }_{\text {dev }}=d e v_{1} \wedge d e v_{2} \wedge \ldots \wedge d e v_{n 1} \\
& a_{\text {sinl }}=\frac{\sum_{l=1}^{l=m_{1}} i n l_{m_{1}}}{m_{1}} * a s e_{d e v} \\
& a^{a s e_{1}}=\lambda_{\text {dev }} * a s e_{d e v}+\lambda_{i n l} * a s e_{i n l} \\
& \text { and } \lambda_{\text {dev }}+\lambda_{\text {inl }}=1
\end{aligned}
$$

In formula(1-6): $d e v_{i}=1$ indicates exist, $\operatorname{dev}_{i}=0$ indicates absent; $i n l_{l=1}$ be suitable for hardware working, inl $_{l=0}$ be not suitable for hardware working; $\lambda_{d e v}$ weight of hardware, $\lambda_{\text {inl }}$ weight of influence factor ; $a s e_{1}$ evaluation result of system hardware class.

B. Basic system software functions evaluation method

Based on the system hardware ,basic system functions is the second layer,which is the essential condition of the system application function .Basic system function include data collection ,data communication ,data processing and computation ,data store and management ,graphic function etc.And the performance of system function include system response time,the main load rate,data error,system privilege management etc.

set of basic system function:

$$
S Y S B=\left\{s y s b_{1}, s y s_{2}, \ldots, \text { sys }_{i}, \ldots, \text { sys }_{n_{2}}\right\}(7)
$$

set of the performances:

$$
P E R B=\left\{\text { perb }_{1}, \text { perb }_{2}, \ldots, \text { perb }_{i}, \ldots, \text { perb }_{m_{2}}\right\}(8)
$$

evaluation function as follows:

$$
\begin{gathered}
a_{\text {sys }}=s y s b_{1} \wedge s y s b_{2} \wedge \ldots \wedge s y s b_{n 2} \\
\text { ase }_{p e r b}=\frac{\sum_{l=1}^{l=m_{2}} \operatorname{perb}_{m_{2}}}{m_{2}} * a s e_{\text {sys }} \\
\text { ase }_{2}=\lambda_{\text {sysb }} * a s e_{\text {sys }}+\lambda_{\text {perb }} * a s e_{\text {perb }} \\
\text { and } \lambda_{\text {sysb }}+\lambda_{\text {perb }}=1
\end{gathered}
$$

In formula(7-12), $s y s b_{1}=1$ indicates exist, $s y s b_{1}=0$ indicates absent; $\operatorname{per}_{1}=1$ performance indicators meet the requirements. $\operatorname{per}_{1}=0$ performance indicators not meet the requirements; $\lambda_{\text {sysb }}$ weight of basic system functions, $\lambda_{\text {perb }}$ weight of basic system functions performance; $a^{a s e_{2}}$ evaluation function of basic system function software 。

C. System application function evaluation method 
other system functions include alarm,tabulation and printing, communication and monitoring, dynamic monitoring, remote maintenance, fault diagnosis.essential application functions include low frequency oscillation monitoring and analysis, power disturbance recognition, evaluation of primary frequency generator ,power system model and parameter check. And the performances include requirement of frequency oscillation calculation error ,alarm accuracy rate of low frequency oscillation ,alarm accuracy rate of power disturbance ,alarm accuracy rate of primary frequency generator ,stored time of the primary frequency modulation ,identification accuracy rate of power system model and parameter ,stability ,fault tolerance ,computational efficiency etc.

set of system application function:

$$
A P P=\left\{a p p_{1}, a p p_{2}, \ldots, a p p_{i}, \ldots, a p p_{n_{3}}\right\}
$$

set of the performances:

$$
\text { PERA }=\left\{\text { pera }_{1}, \text { pera }_{2}, \ldots, \text { pera }_{i}, \ldots, \text { pera }_{m_{3}}\right\}
$$

the evaluation function as follows:

$$
\begin{array}{r}
a_{a s p}=\frac{\sum_{i=1}^{i=m_{3}} a p p_{i}}{m_{3}} \\
a s e_{\text {pera }}=\frac{\sum_{l=1}^{l=m_{3}} \text { pera }_{l} * a p p_{i}}{n_{3}} \\
a_{\text {them }}=\lambda_{\text {app }} * a s e_{\text {app }}+\lambda_{\text {pera }} * \text { ase } e_{\text {pera }} \\
\lambda_{\text {app }}+\lambda_{\text {pera }}=1
\end{array}
$$$$
\text { Among them }
$$

In formula (13-18) : ${ }^{a p p_{i}=1}$ the function existing, $a p p_{i}=0$ the function not existing. pera $_{i=1}$ performance indicators meet the requirements, pera $_{i}=0$ performance indicators not meet the requirements; $\lambda_{\text {app }}$ weight of system application, $\lambda_{\text {pera }}$ weight of system application performance。In formual (16), pera $a_{l}$ is performance of $a p p_{i}$, one ${ }^{a p p_{i}}$ may corresponding to several pera ${ }_{l}$; $a_{3} e_{3}$ is performance of other system function and application function.

D. Whole WAMS system evaluation method

Based on system hardware,system software and application software,evaluation method of the whole WAMS system is designed, in order to evaluate the state and performance of the system.

evaluation function of single time:

$$
\text { ase }_{\text {wams }}=a_{1 s e_{1}}+a_{2 s} e_{2} a_{\text {dev }}+a s e_{3} * a s e_{\text {sys }}
$$

In formula (19), ase $_{\text {wams }}$ evaluation function of whole system.in view of, WMAS is still in primary stage, set $\lambda_{\text {dev }}, \lambda_{\text {sysb }}$ equal to $0.8, \lambda_{\text {inl }}, \lambda_{\text {perb }}$ equal to 0.2 , $\lambda_{\text {app }}$ equal to $0.5, \lambda_{\text {pera }}$ equal to 0.5 。

In order to evaluate stability and reliability of WAMS,continuity several evaluation method is designed,and the function as follows:

$$
\begin{gathered}
p r o_{d e v}=\frac{\sum_{k=1}^{k=n} a s e_{d e v_{-} k}}{n} \\
p r o_{s y s}=\frac{\sum_{k=1}^{k=n} a s e_{s y s_{-} k}}{a v e_{a p p}}=\frac{\sum_{k=1}^{k=n} a s e_{3_{-} k}}{\sum_{k=n} a s e_{\text {wams } k} * a s e_{s y s \_} k} \\
\sum_{k=1}^{k=n} a s e_{s y s_{-} k}
\end{gathered}
$$

In formula(20-23): $\quad$ pro $_{d e v}$ availability rate of system hardware for a period of time; $\mathrm{pro}_{\text {sys }}$ availability rate of basic system function software for a period of time; $a_{\text {avp }}$ integrality and performance of system application function for a period of time;

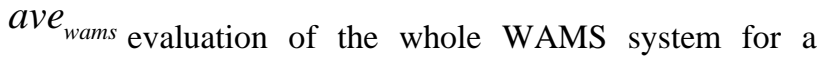
period of time; $k$ is the number of evaluation。

\section{CONCLUSION}

Aim at the operation state of WAMS master station system,the system evaluation is divide into three parts,system hardware,basic system software,other system software and application,and evaluation method and function of every part is designed.At last,based on the three pasts' relation,evaluation method and function of whole WAMS system is designed.For the stability and reliability, continuity several time evaluation function of the system is designed for a period of time,and a quantitative index is computed.

\section{REFERENCES}

[1] Gao Xiang. The grid dynamic monitoring system application technology[M].BeiJing:China Electric Power Press,2011.7.

[2] $\mathrm{Xu}$ Shukai, Xie Xiaorong, Xin Yaozhong. Present application 
situation and development tendency of synchronous phasor measurement technology based wide area measurement system [J]. Power System Technology, 2005, 29(2):44-49.

[3] Wu Jingtao, Xie Xiaorong, Wang Liding, et al. Development and prospect of WAMS in power system. Electrical Equipment, 2006, 7(3):46-49.

[4] Xue Yusheng, Xu Wei, Zhaoyang Dong, et al. A review of wide area measurement system and wide area control system[J], Automation of Electric Power Systems, 2007, 31(15):1-5.

[5] Xue Yusheng, Li Haifeng. A review of CIGRE 2008 on power system operation and control[J]. High voltage Engineering, 2008, 34(11):2253-2258.

[6] He Jiang, Zhou Jingyang, Wang Mingjun. Application of wide area phasor measurement technology in smart grid [J]. Power System Technology, 2009, 33(15):16-19.

[7] Li Qiang, Yu Erkeng, Lu Shichao, et al. An improved optimal PMU placement algorithm [J]. Power System Technology, 2005, 29(12):57-61.

[8] Zhou Jie, Ding Xiaohua, Sun Guocheng, et al. New distributed PMU substation system [J]. Automation of electric power system, 2009, 33(17): 105-108.

[9] Zhang Sheng, Wang Jian, He Chun. Evaluating standard and test Method on function of phasor measurement unit. Automation of
Electric Power systems, 2007, 31(21):102-105.

[10] Mai Ruikun, He Zhengyou, Bo Zhiqian, Research on synchrophasor estimation algorithm based on taylor expansion[J]. Automation of electric power system, 2008, 32(12): 22-26.

[11] Tang Yong. Fundamental theory of forced power oscillation in power system [J]. Power System Technology, 2006, 30(10):29-33.

[12] Xue Yusheng, Hao Sipeng, Liu Junyong, et al. A review of analysis methods for low-frequency oscillations [J]. Automation of Electric Power Systems, 2009, 33(3):1-8.

[13] Song Xiaona, Bi Tianshu, Wu Jingtao, et al. Study on WAMS based power system disturbance identifying method[J]. Automation of electric power system, 2006,30(5):24-28.

[14] Qin Xiaohui, Bi Tianshu, Yang Qixun. WAMS based power system disturbance identification and location approach. Power System technology, 2009, 33(12):35-41.

[15] Zheng Tao, Gao Fuying, On-line monitoring and computing of unit PFR characteristic parameter based on PMU[J]. Automation of electric power system, 2009,33(11):57-61.

Author brief introduction:

Zhao Kun(1984-),male,master,engineer,research on power system and Automation,WAMS.zhaokunfx@126.com. 